\title{
Simulación y evaluación de un proceso productivo de suelas termoplásticas en Colombia ${ }^{1}$
}

\section{Simulation and evaluation of a production process for thermoplastic soles in Colombia}

\author{
A. P. Correa, J. A. Castro, C. Garcés y Y.F. Ceballos \\ Recibido: junio 17 de 2018 - Aceptado: noviembre 16 de 2020
}

\begin{abstract}
Resumen-La industria del calzado está conformada por diversos tipos de productos y es un renglón importante de la economía en Colombia. Los productos de calzado orientadas a mejorar la seguridad y la salud en el trabajo deben cumplir con altos estándares de calidad y su producción es costosa. La producción de suelas termoplásticas para calzado es compleja y es necesario evaluar diferentes fases del proceso que se puedan mejorar y posteriormente aplicar políticas que contribuyan al mejoramiento de estos puntos críticos. Para esta investigación se analizaron las principales variables asociadas a la elaboración de suelas termoplásticas, se identificó dónde estaban ubicados los cuellos de botella y se proponen un conjunto de escenarios de solución, los cuales permiten una reducción en los costos del proceso y en el tiempo de elaboración de productos.
\end{abstract}

Palabras clave - Manufactura, Optimización, Producción Simulación discreta, Simul8®, Suelas termoplásticas, Zapatos.

Abstract - The footwear industry is made up of various types of products and is an important economy line in Colombia. Footwear products aimed at improving occupational safety and health must meet high quality standards and are expensive to produce. The thermoplastic soles production for footwear is

1 Producto derivado del proyecto de investigación "Propuesta de mejoramiento para el proceso productivo de suelas termoplásticas en la ciudad de Medellín.”. Presentado por el Grupo de Investigación Ingeniería y sociedad, de la Universidad de Antioquia.

A. P. Correa, Ingeniera Industrial, Universidad de Antioquia, Medellín, Colombia. email: angiepcorreas@gmail.com

J. A. Castro, Ingeniero Industrial, Universidad de Antioquia, Medellín, Colombia. email: juliocastro1297@gmail.com

C. Garcés, Ingeniera Industrial, Universidad de Antioquia, Medellín, Colombia. email: carolina.garcesj@udea.edu.co

Y.F. Ceballos, PhD en Ingeniería. Profesor Ingeniería Industrial, Universidad de Antioquia, Medellín, Colombia, email:

yony.ceballos@udea.edu.co

Como citar este artículo: Correa, A. P., Castro, J. A., Garcés, C. y Ceballos, Y.F. Simulación y evaluación de un proceso productivo de suelas termoplásticas en Colombia, Entre Ciencia e Ingeniería, vol. 14, no. 28, pp. 10-16, julio-diciembre 2020. DOI: https://doi.org/ 10.31908/19098367.1850.

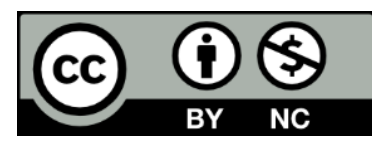

Attribution-NonCommercial 4.0 Intenational (CC By-NC 4.0) complex and it is necessary to evaluate different phases of the process that can be improved and subsequently apply policies that contribute to the improvement of these critical points. For this research, the main variables associated with the elaboration of thermoplastic soles were analyzed, where the bottlenecks were located and a set of solution scenarios are proposed, which allow a reduction in production cost and time.

Keywords - Optimization, Production, Simulation, Simul8®, Manufacturing, Shoes, Thermoplastic soles.

\section{INTRODUCCIÓN}

T A producción de calzado a nivel mundial es una industria Lcreciente [1], [2], y en el último año el sector del calzado en Colombia ha tenido un aumento significativo, principalmente en las exportaciones por más de 7,9 millones de dólares en calzados cuyas suelas son de caucho y termoplástico. Este último material ha incrementado la producción debido a que es uno de los tipos de suelas más apetecidas por su gran variedad de estilos, ya que es un material que puede fundirse a altas temperaturas y permite darle diversas formas y colores, dando un valor añadido al calzado.

Normalmente, las empresas de suelas cuentan con máquinas de termoplástico para llevar a cabo el proceso de inyección y poder dar forma a las suelas de este material a través de moldes de aluminios, y si bien este subproceso es en gran parte automatizado, los subprocesos posteriores como el pulido, pintura, cepillado y empaque son manuales dando lugar a cuellos de botella y a que las empresas tengan que realizar un análisis detallado para equilibrar el componente automatizado con el manual, de forma que los tiempos de entrega del producto final no se vean afectados por demoras y la relación con los clientes no se deteriore [3].

Por lo anterior, es necesario analizar de qué forma se puede modificar el proceso de manera que exista un equilibrio entre cada uno de los subprocesos (manual y automatizado). Para ello puede utilizarse una herramienta como la simulación de eventos discretos, la cual mediante el desarrollo de un modelo acorde a la realidad puede proveer información esencial sobre 
problemas que estén presentes en el proceso y a la vez permite evaluar alternativas en dicho modelo analizando su comportamiento y ayudando a la solución de los problemas reales.

\section{ANTECEDENTES}

Diversas investigaciones permiten identificar que es necesario realizar una evaluación de la forma en la cual se desarrolla el proceso de elaboración de zapatos. En [4] se evidencia que la especialización en un clúster de producción de calzado es necesaria y se concluye que el desarrollo en países de américa latina puede darse en función de esta industria, permitiendo que una gran cantidad de personas puedan tener ingresos y que exista una rápida adaptación en un mercado cambiante. Un trabajo similar en la industria de calzado se presenta en [5] y se busca determinar la política de producción óptima sobre los posibles modelos de zapatos a producir en un día de trabajo aplicando la simulación para ver en qué grado las variaciones de los modelos afectan la tasa de rendimiento de la línea de producción.

Por otro lado, nuevas herramientas tecnológicas desafían la forma como se elabora el calzado actualmente. En [6] se describe el avanzado estado de la automatización en esta industria, donde si bien todavía hay partes de un zapato que son elaboradas manualmente, partes como las suelas son fabricadas en su mayoría mediante el moldeo en máquinas, por ejemplo, de termoplástico o poliuretano. Sin embargo, todavía existen pequeños fabricantes que con procesos no automatizados continúan haciendo frente a la competencia en el mercado del calzado. Algo similar a lo anterior se describe en [7] donde se menciona la importancia de la innovación y la evolución tecnológica en las empresas dedicadas a la producción del calzado, haciendo también hincapié en la capacitación del personal para lograr una estandarización del proceso, esto con el objetivo de lograr mayor competitividad. En [8] de igual forma, se plantea que es necesario establecer un equilibrio entre las actividades manuales y automatizadas para tener un control del proceso y que éste fluya de la manera adecuada; esto se hace con una simulación en donde se examinan los efectos de la mano de obra sobre el proceso.

\section{METODOLOGÍA}

Para describir y analizar el proceso de elaboración de suelas de termoplástico, se comienza con la formulación del problema donde se identifican las necesidades y condiciones específicas del proceso, las cuales hacen necesario este análisis, en esta etapa también se lleva a cabo la recopilación y el modelamiento de datos. Posteriormente, se desarrolla la conceptualización del modelo en donde se construye el modelo computacional, se realiza la validación y verificación de éste con algunas técnicas descritas posteriormente que tienen como fin comprobar que el comportamiento del modelo computacional se relacione con la realidad, adicionalmente, se hace un análisis de sensibilidad con el objetivo de comprobar que el modelo sea robusto. En último lugar, se realiza el diseño de experimentos con el cual se espera hallar una posible solución a los problemas planteados en el modelo, para ello se elaboran posibles escenarios seguido de un análisis de cada uno de éstos que determinará cuál es el mejor escenario para las condiciones especificadas. En la fig. 1 se presenta en esquema que resume la metodología descrita.

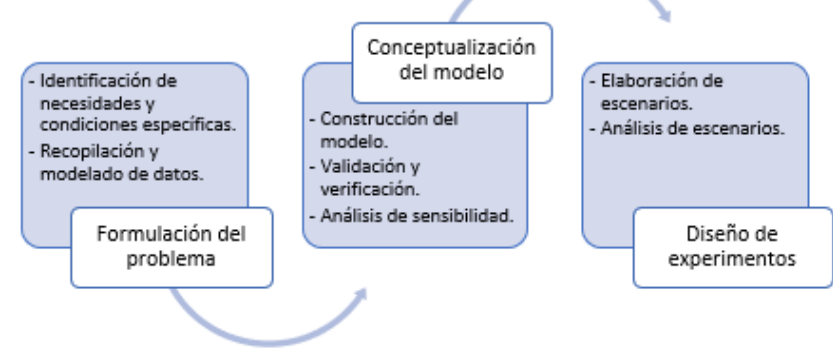

Fig. 1. Metodología empleada en problemas de simulación.

\section{A. Formulación del problema}

Para contextualizar el proceso, en la fig. 2 se presenta la representación gráfica a manera de diagrama de flujo donde se identifican las diferentes etapas y actividades del proceso de elaboración de suelas termoplásticas.

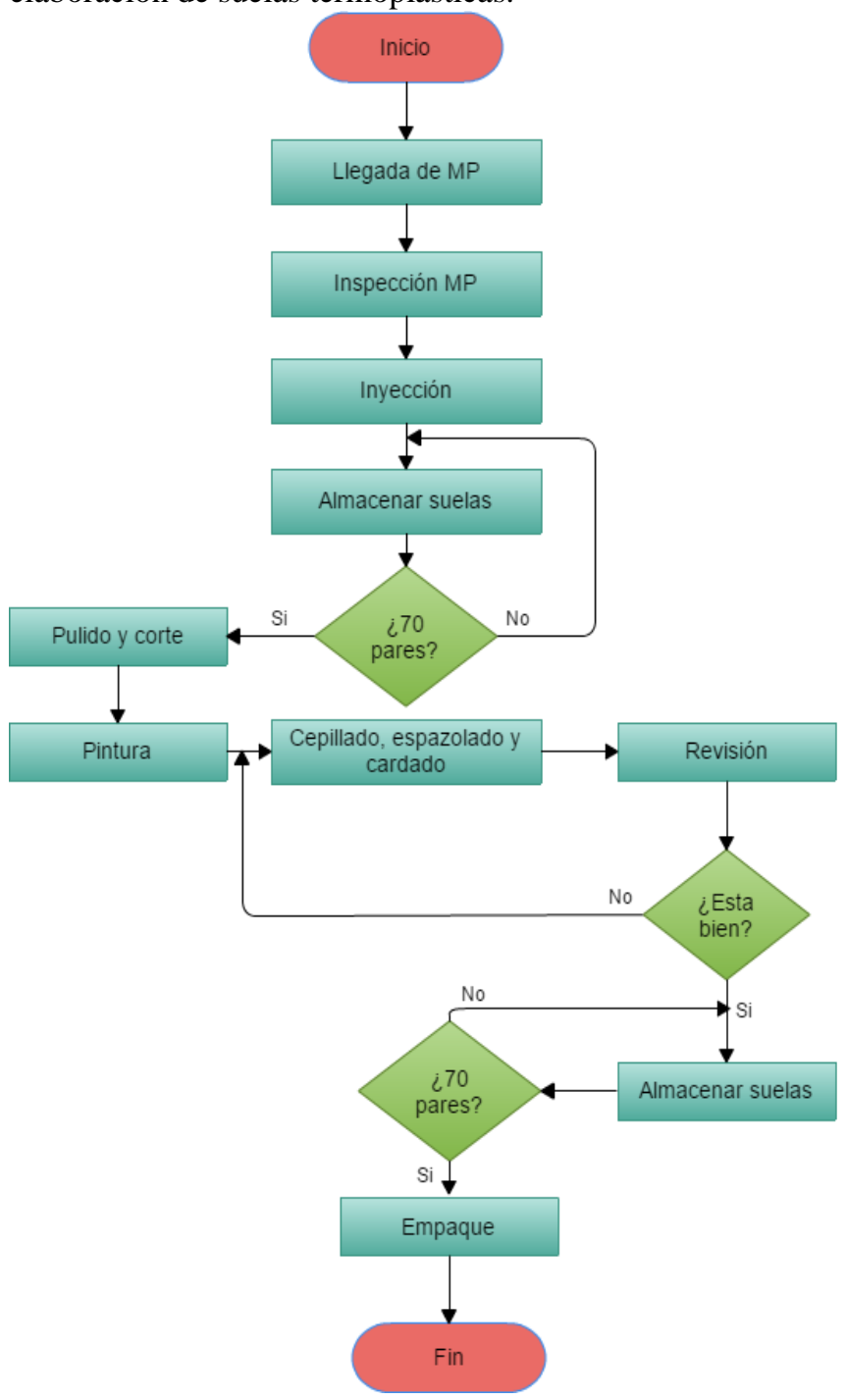

Fig 2. Flujograma del modelo. 
Uno de los principales problemas que se observó, fue los grandes arrumes que se forman en las actividades manuales debido a que la primera parte del proceso es muy automatizada. Uno de los primeros procesos es el de inyección y éste es automatizado, pues las máquinas termoplásticas dan forma a las suelas mediante moldes diseñados previamente y pocos empleados son encargados de éste, porque sólo deben quitar las suelas ya moldeadas por la máquina. Desde este punto hasta que las suelas son finalmente empacadas todo es manual, así que se forman grandes colas de pares de suelas esperando ser manipuladas en el resto de las actividades. Todas estas demoras en cada uno de los servicios hacen que en muchos casos no se pueda cumplir con el tiempo de entrega de los pedidos a los clientes teniendo que pactar nuevos plazos de despacho.

\section{B. Recopilación y modelado de datos}

Durante el modelamiento del proceso de producción de suelas termoplásticas se tuvieron en cuenta las variables descritas en la tabla I que fueron identificadas a partir de una observación realizada al proceso real.

TABLA I.

VARIABLES DEL MODELO.

\begin{tabular}{|c|c|}
\hline \multirow{2}{*}{$\begin{array}{l}\text { Variables } \\
\text { Exógenas }\end{array}$} & Número de pares de suelas pedidas \\
\hline & $\mathrm{Kg}$ de materia prima entrantes \\
\hline \multirow{4}{*}{$\begin{array}{l}\text { Variables } \\
\text { Endógenas }\end{array}$} & Número de máquinas disponibles \\
\hline & Número de operarios disponibles \\
\hline & Proporción de defectuosos de pares de suelas \\
\hline & Proporción de defectuosos de materia prima \\
\hline \multirow{3}{*}{$\begin{array}{l}\text { Variables de } \\
\text { estado }\end{array}$} & Número promedio de pares de suelas en cola \\
\hline & Número promedio de pares de suelas en el sistema \\
\hline & Porcentaje promedio de ocio de empleados \\
\hline \multirow{2}{*}{$\begin{array}{l}\text { Variables de } \\
\text { desempeño }\end{array}$} & Cantidad promedio de pares de suelas defectuosas \\
\hline & Porcentaje promedio de ocio de los empleados \\
\hline
\end{tabular}

Posteriormente, se ajustaron las variables asociadas al modelo utilizando el software @Risk y los resultados de dichos ajustes se encuentran a continuación en la Tabla II.

TABLA II.

AJUSTE DE DISTRIBUCIONES

\begin{tabular}{|l|l|l|}
\hline Variable & $\begin{array}{l}\text { Tipo de } \\
\text { distribución }\end{array}$ & Parámetros \\
\hline Tiempo de inspección & Exponencial & Media $=3,84138$ \\
\hline $\begin{array}{l}\text { Tiempo de inyección } \\
\text { maquina 1 }\end{array}$ & Weibull & $\begin{array}{l}\text { Alpha }=2,4805 \\
\text { Beta }=3,3006 \\
\text { Desplazamiento }=3,6265\end{array}$ \\
\hline $\begin{array}{l}\text { Tiempo de inyección } \\
\text { maquina 2 }\end{array}$ & Weibull & $\begin{array}{l}\text { Alpha }=1,5934 \\
\text { Beta }=0,9599 \\
\text { Desplazamiento }=4,4537\end{array}$ \\
\hline $\begin{array}{l}\text { Tiempo proceso de } \\
\text { pulido }\end{array}$ & Triangular & $\begin{array}{l}\text { Mínimo }=1,2428 \\
\text { Más probable }=1,789 \\
\text { Máximo }=2,92\end{array}$ \\
\hline $\begin{array}{l}\text { Tiempo proceso de } \\
\text { pintura }\end{array}$ & Uniforme & $\begin{array}{l}\text { Mínimo }=1,1418 \\
\text { Máximo }=3,7917\end{array}$ \\
\hline $\begin{array}{l}\text { Tiempo proceso de } \\
\text { cepillado, espazolado y } \\
\text { cardado }\end{array}$ & Normal & $\begin{array}{l}\text { Media }=2,45 \\
\text { Desviación estándar }=0,3572\end{array}$ \\
\hline $\begin{array}{l}\text { Tiempo proceso de } \\
\text { inspección } \\
\text { de suelas }\end{array}$ & Triangular & $\begin{array}{l}\text { Mínimo }=0,9877 \\
\text { Más probable }=0,9877 \\
\text { Máximo }=3,3056\end{array}$ \\
\hline $\begin{array}{l}\text { Tiempo proceso de } \\
\text { empaquetado }\end{array}$ & Normal & $\begin{array}{l}\text { Media }=1,9769 \\
\text { Desviación estándar }=0,9136\end{array}$ \\
\hline
\end{tabular}

\section{Elaboración de un modelo formal}

Para la elaboración de un modelo formal que representara el proceso de elaboración de suelas termoplásticas, se utilizó el software Simul8®, donde se tuvo en cuenta la conceptualización del modelo y la descripción realizada anteriormente, al mismo tiempo que se emplearon las distribuciones ajustadas previamente para los diferentes centros de trabajo. El proceso detallado es descrito a continuación.

A la empresa llegan pedidos de pares de suelas termoplásticas según una distribución average con media de 1,36433 minutos (es decir, llega un par de suelas como pedido), después estos pedidos pasan a una cola de pedidos mientras la materia prima llega (termoplástico) y pueda ser transformada. Para este modelo, se asume que la entrada de materia prima es infinita, ya que la empresa nunca ha tenido ningún tipo de inconveniente con los proveedores en cuanto a disponibilidades de ésta.

Posteriormente, la materia prima entrante es inspeccionada por dos operarios bajo una distribución triangular con un valor mínimo de 1,9665 , valor más probable y máximo iguales a 2,78; además según datos suministrados por la empresa, el $20 \%$ de materia prima no cumple con las especificaciones y es devuelta a los proveedores, por lo que este porcentaje se convierte en una salida en el modelo.

Luego, la materia prima es almacenada para luego pasar a la inyección donde los pedidos de pares de suela comienzan a ser atendidos. Existen dos máquinas de inyección, cada una con capacidad para producir 3 pares de suelas y cada par de suelas necesita de $0,33 \mathrm{~kg}$ de termoplástico para que su molde quede hecho, luego, cada máquina al procesar 3 pares de suelas al mismo tiempo necesita ser cargada con $1 \mathrm{~kg}$ de materia prima; ambas máquinas trabajan bajo la distribución Weibull, pero con parámetros diferentes. En este proceso de inyección están involucrados 2 empleados, 1 por cada máquina.

Posterior a la inyección, las suelas son almacenadas hasta que en total haya 70 pares de suelas en almacén y luego son llevadas a pulido y corte donde esperan para que se les realice este proceso que se distribuye triangularmente con un valor mínimo de 1,2428; un valor medio de 1,789 y un valor máximo de 2,92 (todos en minutos) para los dos centros de trabajo dispuestos en esta actividad y son necesarios 2 operarios para ello.

Una vez pulidos los pares de suelas, pasan a esperar por ser pintados por un operario que trabaja bajo una distribución uniforme con un valor mínimo de 1,1418 y un máximo de 3,7917 minutos. Cada par de suelas se somete al cepillado, cardado y espazolado por alguno de los dos operarios encargados de dicha función que con la ayuda de una cepilladora y cardadora completa esta acción con una distribución normal de media 2,45 minutos y una desviación estándar de 0,3572 y posteriormente pasan a la cola de espera de inspección.

Los pares de suelas esperan a ser inspeccionados bajo una distribución y según estimaciones de la empresa, cerca del $10 \%$ de los pares de suelas tienen imperfecciones y necesitan 
volver al cepillado, cardado y espazolado. Aquellos parees (suelas que no tienen ningún tipo de imperfección pasan a ser almacenados hasta completar 70 pares, como en el proceso de inyección, para ser empacados.

El empaque es realizado por dos personas y según los datos tomados por los investigadores encargados de esta modelación, esta actividad se distribuye de manera normal con una media de 1,9769 minutos y una desviación estándar de 0,9136 y de esta manera los pares de suelas quedan listos para ser entregados al cliente.

El sistema se modela desde las 8:00 am hasta las 6:00 para un total de 10 horas de trabajo diarias y teniendo en cuenta que la empresa labora 5 días a la semana. El tiempo de simulación decidido por los investigadores es de 1 semana, tiempo considerado suficiente para realizar conclusiones según los modeladores de este proceso.

\section{Verificación y validación}

La verificación es un elemento fundamental en la simulación de sistemas discretos, dado que es el proceso por el cual se comprueba que la lógica del modelo computacional corresponda con el modelo conceptual y los requisitos de diseño de éste, es decir, que el modelo sea lo que los diseñadores de éste piensan que es similar al descrito por Izquierdo [9].

Por lo anterior, para la verificación del modelo de simulación realizado se utilizaron técnicas como las trazas de simulación, donde se realiza el seguimiento del flujo de las entidades (pares de suelas, en este modelo) a través de cada sección del modelo y en el modelo completo, con el objetivo de determinar si su comportamiento y lógica son adecuadas. [10]. El software Simul8® permite ver el comportamiento de los pares de suelas a través del sistema. Al realizar la corrida del sistema se concluyó que el comportamiento y dinámica de estas es adecuada, ya que se comporta como se esperaba.

También se hizo la verificación del modelo mediante el método de degradación analizando el comportamiento del modelo computacional en condiciones extremas, esto fue aplicado tanto para los valores de entrada o variables exógenas como para los parámetros y variables internas [10]. Para este método de verificación se procedió a cambiar la distribución de la llegada de pedidos, esta variable de entrada tomó un valor fijo de un pedido por minuto, por lo que, si la estructura del modelo era coherente y confiable se esperaba que, en una jornada de 10 horas, que equivale a un día laboral, llegaran 600 pedidos (10jornadahoras*60minutos) aunque en la realidad en el último minuto no llegan pedidos, así que el valor de la llegada de pedidos debía ser 599.

Con el objetivo de incrementar el nivel de credibilidad y aceptación del modelo se realizó la validación, de esta manera podríamos asegurar que el modelo representaba el comportamiento real del sistema y podía ser utilizado para futuros experimentos cuyos resultados permitirían a las directivas de la empresa tomar decisiones pertinentes (Banks, 2010). Por eso, para averiguar si el modelo producía resultados cercanos a la realidad, era fundamental conocer la opinión de aquellas personas inmersas en el proceso, por lo que la intuición de expertos fue una técnica importante para validar el modelo representado.

El modelo realizado en el software Simul8® fue presentado al encargado de producción y a uno de los gerentes de la empresa. El jefe de producción manifestó que gráficamente se veía un modelo muy parecido a lo que en realidad se da en el proceso. Por otro lado, las grandes colas presentadas en el pulido y pintura efectivamente se hacen en la realidad y de hecho fue uno de los problemas que fue planteado al inicio, ya que estas actividades se desarrollan casi en su totalidad de forma manual mientras que al ser la inyección una actividad automatizada, los pares de suelas salen a mayor velocidad y por ello se estancan antes de los procesos manuales, lo que en verdad produce que los tiempos de entrega de los pedidos de suelas se tengan que extender.

Finalmente, basados en las opiniones de personas expertas en el proceso que se está simulando y en todas las consideraciones descritas previamente, el modelo representado es válido, su comportamiento se aproxima a lo que sucede en el proceso real y permite continuar para analizar de lleno los resultados arrojados.

\section{E. Análisis de sensibilidad}

Para analizar qué tan sensible era el modelo representado en Simul8®, se realizaron cambios en la tasa de llegada de los pedidos, los parámetros del tiempo de cepillado, cardado y espazolado, y el número de suelas que requerían ser almacenadas antes de pasar a pulido y empaque.

Se realizaron entonces dos cambios en la llegada de pedidos, primero teniendo una distribución Average con media de 1 minuto y un segundo cambio con la misma distribución, pero con una media de 2 minutos. Con estos ajustes se observaron variaciones significativas en las colas del pulido y de pintura, sin embargo, no se presentan cambios abruptos en las demás variables tenidas en cuenta para esta modificación.

En otro escenario, se cambió la media de la actividad de cepillado, cardado y espazolado a una de 2 minutos y se observó que los cambios en las variables no son significativos, incluso el número de pares de suelas terminadas en promedio es igual al de la situación inicial. Al incrementar la media a 3 tampoco se observaron mayores cambios.

Por último, se cambió el número de suelas que requerían ser almacenadas antes de pasar a pulido y empaque. Inicialmente, se probó pasando de 70 pares a 50, luego también se probó con 90 pares y los resultados no fueron muy diferentes a los valores originales, tan sólo en el número de pares de suelas terminadas en promedio se ve una pequeña diferencia al igual que en la cola promedio del pulido, pero los cambios no son significativos. Por lo tanto, con base en los cambios analizados el modelo representado en simul8® no mostró cambios fuertes al modificar tasas y parámetros.

\section{Resultados}

Con el sistema ya modelado, verificado y validado, se comenzaron a plantear posibles escenarios para dar solución al problema inicialmente planteado teniendo en cuenta el comportamiento y estado actual del sistema. 


\section{A. Estado actual}

Antes de plantear los posibles escenarios para resolver los problemas y puntos críticos del modelo, es apropiado exponer cuáles son éstos. Para ello, se analizó una simulación de 5 días de 10 horas laborales.

En la simulación se evidenció una cola significativa antes de la tarea de pintura, lo cual hacía que las tareas consecutivas a la pintura no tuvieran material (suelas) para trabajar y el tiempo de ocio se incrementara. Por otra parte, el almacenamiento de los 70 pares de suelas (antes de pulido y corte y de empaque) hacía que se perdiera tiempo mientras se esperaba que éstas fueran recolectadas.

En la Tabla III se observa que 7 de los 11 operarios tenían una utilización inferior o igual al $70 \%$, lo cual se traducía en muchos empleados ociosos y/o innecesarios.

TABLA III.

UTILIZACIÓN PROMEDIO DE LOS OPERARIOS

\begin{tabular}{|c|c|c|}
\hline Tarea & Operario & Utilización promedio \% \\
\hline \multirow[t]{2}{*}{ Inspección } & Operario1 & 100 \\
\hline & Operario2 & 75,72 \\
\hline \multirow[t]{2}{*}{ Inyección } & Operario3 & 25,39 \\
\hline & Operario4 & 10,52 \\
\hline \multirow[t]{2}{*}{ Pulido y corte } & Operario5 & 70,62 \\
\hline & Operario6 & 70,55 \\
\hline Pintura & Operario7 & 96,54 \\
\hline \multirow{2}{*}{$\begin{array}{l}\text { Cepillado, cardado y } \\
\text { espazolado }\end{array}$} & Operario8 & 52,7 \\
\hline & Operario9 & 52,78 \\
\hline \multirow[t]{2}{*}{ Empaque } & Operario10 & 37,21 \\
\hline & Operario11 & 37,17 \\
\hline
\end{tabular}

\section{B. Análisis de escenarios y escenario solución}

Para dar solución a los problemas visualizados en el modelo, se plantearon tres escenarios que fueron simulados en el modelo para observar su comportamiento y posteriormente elegir el escenario solución teniendo siempre en cuenta que dicho escenario no debía ser de compleja implementación para la empresa, así se estaría planteando una solución real y factible.

El escenario 1 tenía como objetivo disminuir la cola de pintura añadiendo un puesto de trabajo en dicha área, ya que teniendo en cuenta que la utilización de los operarios destinados a inyección era muy baja, uno de ellos sería trasladado a la sección de pintura para cubrir el nuevo puesto de trabajo, de esta manera la cola en esta sección sería menor y los operarios en el cepillado, corte, espazolado y empaque tendrían más pares de suelas para trabajar.

Con los cambios aplicados al modelo para el escenario 1 se cumplió con el objetivo de disminuir la cola del proceso de pintura, ésta tuvo una disminución del 98,76\%. El tiempo de ocio de los operarios de los procesos de cepillado, cardado, espazolado y empaque también disminuyó, pero se presentó un aumento significativo en el tamaño de la cola de la inspección 2 debido a que la sección de pintura despacha suelas más rápido y éstas quedan estancadas en el proceso de inspección, el cual sólo cuenta con un operario.

En otro caso, el escenario 2 tenía como objetivo aumentar la utilización de los centros de trabajo bajo el supuesto de no tener que esperar el almacenamiento de 70 pares de suelas luego de la inyección e inspección, ya que esta espera ocasiona que los centros de trabajo permanezcan en ocio una gran proporción de tiempo.

Para analizar los resultados del segundo escenario, se hicieron dos experimentos con respecto a la cantidad de pares de suelas que debían esperar para pasar al siguiente proceso. En el primer caso se redujo a la mitad de los pares de suelas (35 pares) y tal como se esperaba, el porcentaje de utilización aumentó en la mayoría de los procesos, aunque en pequeñas proporciones, por lo tanto, se evaluó la opción de que la empresa no tuviera un requisito de pares de suelas almacenadas para pasar de proceso. Sin embargo, al igual que el experimento anterior, el porcentaje de utilización en los centros de trabajo aumentó, pero muy poco. De esta manera, pudo observarse que la política del número de suelas requeridas en almacenamiento para pasar de un proceso a otro no afectaba el tiempo de producción de cada uno de los procesos.

El último escenario planteado tenía el objetivo de disminuir las colas de pintura e inspección de suelas tomando como supuesto que las colas de dichos subprocesos disminuirían si se añadía un puesto de trabajo a cada una de estas actividades y con esto, el tiempo de ocio de los operarios en el cepillado, cardado, espazolado y empaque también disminuiría. Lo anterior se haría trasladando un operario de inyección a la sección de pintura, ya que la utilización de los operarios destinados a inyección es muy baja y así la cola en pintura sería menor. Por último, un nuevo operario tendría que ser contratado para que se dedicara exclusivamente a la inspección de las suelas.

Aplicar este tercer y último escenario permitió que las colas en el proceso de pintura e inspección disminuyeran significativamente en un $98,76 \%$ y $93,94 \%$ respectivamente. También se encontró que el tiempo de ocio en el proceso de cepillado, cardado y espazolado disminuyó en un $81,41 \%$ y el de pintura un $50,36 \%$.

Con los resultados obtenidos en los escenarios planteados en el diseño de experimentos, se analizó que la empresa de producción de suelas termoplásticas debía realizar las siguientes modificaciones:

Asignar una nueva función a uno de sus empleados de inyección (operario 4), teniendo en cuenta que el tiempo de ocio de éste en la tarea de inyección es del 89,48\%. Este operario también trabajaría en la tarea de pintura, para esto se debe disponer de un nuevo centro de trabajo.

Con el nuevo centro de trabajo se eliminaría la cola en la tarea de pintura y por esto llegarían más pares de suelas a las tareas posteriores (CCE, inspección 2 y empaque) por lo que en la inspección 2 se generaría una nueva cola, dado que solo hay dos operarios destinados a la inspección de materia prima (inspección 1) e inspección de suelas (inspección 2). Para eliminar este cuello de botella se sugiere contratar un nuevo empleado que esté asignado sólo a la sección de inspección de suelas.

\section{CONCLUSIONES}

Inicialmente se pensaba que la política de recolectar 70 pares antes de pasar a los procesos de pulido y empaque 
ocasionaba que hubiese mayor acumulación en todas las actividades manuales, pero a través de uno de los experimentos realizados en el modelo planteado se observó que dicha política no es la causante del problema descrito.

Varios de los operarios tienen un alto porcentaje de ocio y esto es algo que sí ocurre en el proceso real, por lo que algunos de los operarios, especialmente uno de los de inyección quien es el que presenta mayor porcentaje de ocio, deberían asignarse a tareas como la pintura cuya cola de pares de suelas es bastante alta. Sin embargo, hay que aclarar que, si el operario de inyección pasara a pintura, la cola de pintura disminuiría notoriamente, pero la cola de inspección previa al empaque aumentaría bastante, debido a que las suelas luego de la pintura circulan con mayor rapidez y en la inspección de los pares de suelas sólo hay un operario.

Se identifica que lo más adecuado es aumentar un puesto de trabajo en la actividad de pintura que sería ocupado por el operario de inyección con mayor porcentaje de ocio y asî brindaría apoyo a la pintura para disminuir las colas presentadas, sin embargo, con esta modificación las colas en la inspección de pares de suelas previa al empaque se incrementarían por lo que se hace necesaria la contratación de un nuevo operario que estaría encargado exclusivamente de este proceso de inspección (escenario 3) y así durante el proceso no se presentarán colas tan gigantescas, los pares de suelas fluirían de manera más rápida por todo el proceso y no se tendrían que ampliar tanto los plazos de entrega con los clientes.

\section{REFERENCIAS}

[1] M. Gonzalez, "Producción de calzado muestra un crecimiento importante," El tiempo, Bogota D.C., pp. 5-6, 2017.

[2] R. Kleer and F. T. Piller, "Local manufacturing and structural shifts in competition: Market dynamics of additive manufacturing," Int. J. Prod. Econ., vol. 216, pp. 23-34, 2019.

[3] P. Caravaggi, A. Giangrande, G. Lullini, G. Padula, L. Berti, and A. Leardini, "In shoe pressure measurements during different motor tasks while wearing safety shoes: The effect of custom made insoles vs. prefabricated and off-the-shelf," Gait Posture, vol. 50, pp. 232-238, 2016.

[4] H. Schmitz, "Small shoemakers and fordist giants: Tale of a supercluster," World Dev., vol. 23, no. 1, pp. 9-28, 1995.

[5] M. S. Eryilmaz, "Analysis of Shoe Manufacturing Factory By Simulation of Production Processes," Southeast Eur. J. Soft Comput., vol. 1, no. 1, pp. 120-127, 2017.

[6] B. W. Rooks, "Robots bring automation to shoe production," Assem. Autom., vol. 16, no. 3, pp. 22-25, 1996.

[7] L. Martinez, B. Arango, and J. Betancourt, "Implementación de herramientas para el diagnóstico de innovación en una empresa del sector calzado en Colombia," RAI-Rev. Adm. e Inovação, vol. 12, no. 3, pp. 310-329, 1809 .

[8] M. Mori, K. Ota, A. Matsubara, and H. Mizuyama, "Design and formation of workforce skills for machine tool assembly," CIRP Ann., vol. 64, no. 1, pp. 459-462, 2015.

[9] L. R. Izquierdo, J. M. Galán Ordax, J. I. Santos, and R. Del Olmo Martínez, "Modelado de sistemas complejos mediante simulación basada en agentes y mediante dinámica de sistemas," Empiria. Rev. Metodol. ciencias Soc., vol. 0, no. 16, p. 85, 2014.

[10] T. Monleón Getino, "Optimización de los ensayos clinicos de farmacos mediante simulación de eventos discretos, su modelización, validación, verificación y la mejora de la calidad de sus datos," TDX (Tesis Dr. en Xarxa), Jan. 2006.

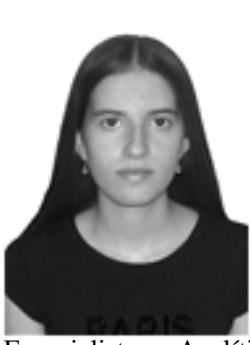

Angie Paola Correa Sepúlveda: Es Ingeniera Industrial de la Universidad de Antioquia. Fue investigadora del Grupo de Investigación INCASInnovación y Gestión de Cadenas de Abastecimiento (Actualmente Grupo ALIADO). Entre sus intereses de investigación se incluyen los modelos de simulación de procesos productivos, la aplicación de métodos cuantitativos y modelos estadísticos predictivos en la industria a partir de la Analítica y Minería de Datos. Desde 2019 se desempeña como Especialista en Analítica de Información en la industria de alimentos. ORCID https://orcid.org/0000-0001-9728-7607

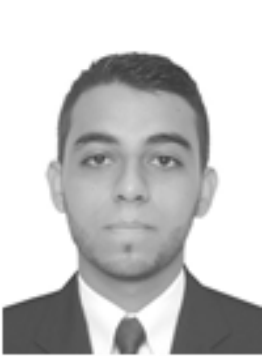

Julián Andrés Castro Castro: Es Ingeniero Industrial de la Universidad de Antioquia. Entre sus intereses de investigación se incluyen modelos de simulación de procesos logísticos, aplicación de métodos cuantitativos y modelos estadísticos predictivos en pro del mejoramiento de procesos en plantas productivas y centros de distribución a partir del análisis de bases de datos. Desde el 2019 se desempeña como Analista de la Planeación de la Demanda en el sector de alimentos. ORCID: https://orcid.org/0000-0003-2706-8462

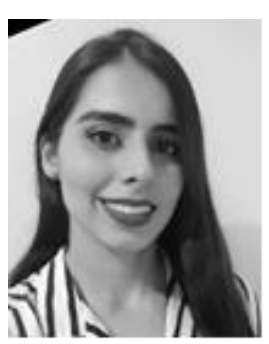

Carolina Garcés Jiménez: Ingeniería industrial de la Universidad de Antioquia con énfasis en finanzas, ha realizado cursos de gestión de indicadores y manejo de inventarios. Ha realizado actividades relacionadas con valuación y diseño de puestos de trabajo, balanceo de línea, establecimiento de estándares y métodos de trabajo, entre otras actividades que ayudan a incrementar la productividad de la empresa. Desde el 2019 se desempeña como Analista en el sector de alimentos. ORCID: https://orcid.org/0000-0003-2156-318X.

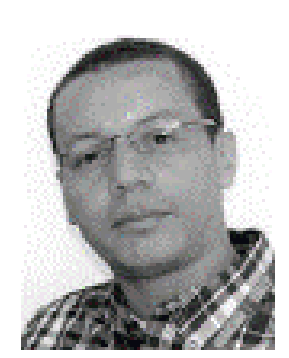

Yony Fernando Ceballos. Se graduó en Ingeniería de sistemas e informática (B.S.), M.S y PhD. en Ciencias de la Computación de la Universidad Nacional de Colombia, Medellín, en 2004, 2007 y 2015, respectivamente. De 2005 a 2013 fue profesor del Departamento de Informática. Desde 2014, es Profesor Asistente del Departamento de Ingeniería Industrial de la Universidad de Antioquia. Sus intereses de investigación incluyen métodos numéricos, teoría de juegos, simulación de sistemas, investigación del comportamiento, algoritmos y procesos estocásticos. Actualmente su investigación se centra en modelos de simulación y aplicación de métodos cuantitativos en problemáticas sociales. El Dr. Ceballos es miembro actual de la Society for Industrial and Applied Mathematics (SIAM) and the system dynamics society (SDS). ORCID: https://orcid.org/0000-0001-5787-8832 